\title{
Changes in the collagenous matrix of the aging human lamina cribrosa
}

\author{
Julie Albon, Wojciech S S Karwatowski, Nicholas Avery, David L Easty, Victor C Duance
}

\begin{abstract}
Aims-The age-related changes in the biochemical composition of the collagenous matrix of the human lamina cribrosa were investigated.

Methods-An age range (3 weeks to 92 years old) of human laminae cribrosae, dissected free of any surrounding structures which contained collagen, were analysed for collagen solubility $(n=58)$ total collagen content $(n=46)$, proportion of collagen types $(n=38)$, and collagen cross linking $(n=30)$, using hydroxyproline analysis, scanning densitometry of peptides after cyanogen bromide digestion, and high performance liquid chromatography, respectively.

Results-Age-related changes included an increase in total collagen and a decrease in the proportion of type III collagen within the lamina cribrosa. The collagen cross link pyridinoline was present at low levels, but demonstrated no trend with age. An age-related increase was found in pentosidine, an advanced glycation product.

Conclusion-These changes in collagen composition imply that the mechanical properties of the lamina cribrosa are altered, resulting in a stiffer, less resilient structure with age. Such alterations in structure may contribute to the increased susceptibility of the elderly to axonal damage in chronic open angle glaucoma.

(Br F Ophthalmol 1995; 79: 368-375)
\end{abstract}

Chronic open angle glaucoma (COAG) is an optic neuropathy characterised by axonal damage and retinal ganglion cell death, accompanied by optic disc cupping and a characteristic pattern of visual field loss. COAG is frequently associated with an increased intraocular pressure although low tension glaucoma is a well recognised condition. ${ }^{1}$ The causes of glaucoma whether genetically or environmentally determined appear to be multifactorial.

Theories as to the pathogenesis of primary open angle glaucoma can be divided between those that invoke the direct and indirect effects of intraocular pressure and those that invoke a vasogenic cause. ${ }^{2}$ The structure of the lamina cribrosa may be particularly relevant in the former case.

There is significant evidence that raised intraocular pressure is important in the development of glaucomatous optic nerve damage. ${ }^{3}$
The regional differences in the architecture of the lamina cribrosa, with the greater pore size and less dense connective tissue in the superior and inferior regions of the lamina cribrosa ${ }^{4}$ appear to correlate with the development of inferior and superior arcuate field loss in glaucoma and the relative preservation of nasal and temporal fibres representing temporal and central field loss, respectively. ${ }^{6}$

Changes observed in the lamina cribrosa in COAG include compression of the lamina plates, posterior rotation of the peripheral lamina cribrosa, ${ }^{7}$ and elongation of the lamina pores. $^{8}$

Immunohistochemical and electron microscopy studies of the connective tissue components in normal, ${ }^{910}$ aging, ${ }^{11}$ and glaucomatous ${ }^{12-14}$ eyes, have identified types $I$, III, IV, V, and VI collagen and elastin in the lamina cribrosa. Studies on numerous tissues have shown collagen types I and III to be the major structural fibrillar components of stromal extracellular matrices. Type IV collagen is a ubiquitous component of basement membranes and the filamentous types $\mathrm{V}^{15}$ and $\mathrm{VI}^{16}$ connect the fibrillar collagens to surrounding connective tissue structures. Therefore the components which constitute the extracellular matrix of the lamina cribrosa provide an essential complementary supportive structure.

Age is an important risk factor in glaucoma. ${ }^{17}$ During the aging process, collagen types I, III, IV, and V, and the thickness of elastic fibres appear to increase within the cores of the cribriform plates. ${ }^{11}$

In glaucoma, type VI collagen increases within the cribriform plates and the basement membranes extend into the nerve bundles. ${ }^{12} \mathrm{~A}$ decrease in fibrillar collagens ${ }^{13}$ and disorganisation of elastic fibres within the core of the cribriform plates and loss at the region of insertion, together with an accumulation of bundles of microfibrils also occurs. ${ }^{14}$

Changes in the support matrix of the lamina cribrosa will alter its mechanical properties and therefore its ability to support the axons. In aging and COAG, compositional alterations in structure may contribute to optic disc cupping and susceptibility to nerve fibre damage which results in visual field loss and eventual blindness.

No attempt has been made to quantitate the changes in collagen which may predispose elderly eyes to glaucomatous damage. Therefore the present study was undertaken to determine and quantify the biochemical changes of collagen in the lamina cribrosa with age. 


\section{Materials and methods}

SOURCE OF TISSUE

Human cadaver eyes, encompassing an age range from 3 weeks to 92 years, were collected within 48 hours of death. All eyes were received from the Corneal Transplant Service Eye Bank at Bristol Eye Hospital after corneas had been removed for transplantation.

\section{ISOLATION OF LAMINA CRIBROSA}

\section{Immunolocalisation of collagen types}

Immunolocalisation of collagen types I, II, III, IV, V, and VI was carried out to determine their distribution in the lamina cribrosa, its central retinal artery and vein, and the surrounding sclera.

Following rapid freezing in isopentane, cooling in liquid nitrogen, and embedding in Tissue-Tek, $8 \mu \mathrm{m}$ thick sections were cut from optic nerves, at the level of the lamina cribrosa using a cryostat. The sections were digested overnight at room temperature with chondroitinase ABC (1 unit $/ \mathrm{ml}$ ) in $0.25 \mathrm{M}$ enriched TRIS- $\mathrm{HCl}(2 \cdot 4 \%$ sodium acetate, $1.46 \% \mathrm{NaCl}, 0.005 \%$ bovine serum albumin) pH 8.0 containing the following protease inhibitors: $100 \mu \mathrm{M}$ EDTA, $10 \mu \mathrm{M} N$-ethylmaleimide (Sigma), $10 \mu \mathrm{M}$ phenylmethyl sulphonyl fluoride (Sigma), and $1.5 \mu \mathrm{M}$ pepstatin (Sigma).

The sections were washed in phosphate buffered saline (PBS) and were incubated for 2 hours with specific antibodies raised against human collagen types I, II, III, IV, V (Southern Biotechnology Associates Inc), and type VI (Bioquote), or non-immune serum as a control. Following washing in PBS (three times), the sections were incubated for 2 hours with appropriate fluorescein conjugated antibodies (Sigma). After extensive washing with PBS the sections were mounted in Citifluor (Agar Ltd) and viewed using a Leitz Dialux microscope with epifluorescence and a Bio-Rad MRC 500 confocal laser scanning microscope (CLSM).

\section{Dissection procedure}

Having demonstrated the distribution of collagen types in the lamina cribrosa, the central retinal vessels, and surrounding sclera a method was developed to dissect the lamina cribrosa away from the adjacent tissues which would contribute to biochemical analyses with their own collagen content (see results).

\section{COLLAGEN CONTENT}

Dissected lamina cribrosa were freeze dried and their dry weight recorded. Following delipidation in 3:1 chloroform:methanol, the tissue was rinsed in distilled water, freeze dried, and then hydrolysed in $6 \mathrm{M}$ hydrochloric acid $(\mathrm{HCl})$ at $110^{\circ} \mathrm{C}$ for 24 hours. The hydrolysates were analysed for the presence of hydroxyproline using a Chemlab autoanalyser by the method of Bannister and Burns. ${ }^{18}$

\section{QUANTITATION OF TYPE I AND TYPE III}

\section{COLLAGEN}

Laminae cribrosae were freeze dried, delipidated, and digested with cyanogen bromide $(\mathrm{CNBr})$ by the method of Light. ${ }^{19} \mathrm{CNBr}$ digestion was carried out in $70 \%$ formic acid at room temperature for 24 hours. A 10-fold dilution with deionised water halted the reaction and the solubilised collagen peptides were freeze dried, dissolved in sodium dodecyl sulphate (SDS) sample buffer, and separated by SDS-polyacrylamide gel electrophoresis according to the method of Laemmli. ${ }^{20}$ The samples were denatured at $60^{\circ} \mathrm{C}$ for 30 minutes and the peptides were then electrophoresed at $35 \mathrm{~mA}$ per gel using a $12.5 \%(\mathrm{w} / \mathrm{v})$ SDS-polyacrylamide slab gel and a $4 \cdot 25 \%$ stacking gel. The gel was stained in $0.005 \%$ Coomassie brilliant blue $\mathbf{R}$ (Sigma) in $10 \%$ methanol, $7 \cdot 5 \%$ acetic acid in water for 24 hours, and then destained in $10 \%$ methanol, $7 \cdot 5 \%$ acetic acid in water for 24 hours. The gels were scanned using a LKB ultrascan XL enhanced laser densitometer (Bromma, Sweden) and the peak areas of the relevant peptides $\alpha 1$ (I)CB8 and $\alpha 1$ (III)CB5 were traced using a computer linked visual imaging display system (VIDS). Percentage type I and type III collagen was calculated taking into account the molecular weight of each peptide and the number of the relevant $\alpha$ chains per molecule. ${ }^{21}$

\section{SOLUBILISATION OF COLLAGEN}

Freeze dried laminae cribrosae were delipidated as above and treated with dithiothreitol (DTT) in $0 \cdot 1 \mathrm{M}$ TRIS buffer $\mathrm{pH} 8.0$ containing $50 \mathrm{mM} \mathrm{NaCl}$ and $5 \mathrm{mM} \mathrm{MgCl}$ for 1 hour at room temperature. After rinsing in deionised water, collagen was solubilised using $\mathrm{CNBr}$ as above. ${ }^{19}$ The undigested tissue containing insoluble collagen was separated from the solubilised collagen in the acid fraction and both fractions were freeze dried to remove excess $\mathrm{CNBr}$ and acid. The fractions were then hydrolysed and analysed for hydroxyproline as above. 18

\section{ANALYSIS OF COLLAGEN CROSS LINKS}

There are two major groups of collagen cross links: those initiated by the enzyme lysyl oxidase and those derived from non-enzymatic glycosylation of lysine and hydroxylysine residues. Two major products in mature connective tissues include hydroxylysyl pyridinoline (HP) and pentosidine respectively.

Freeze dried, delipidated laminae cribrosae were hydrolysed in $6 \mathrm{M} \mathrm{HCl}$ as above. After freeze drying, the hydrolysate was rehydrated in $1 \mathrm{ml}$ of deionised water. An aliquot was taken for hydroxyproline analysis ${ }^{17}$ to determine amount of collagen in the sample and the remainder was freeze dried. Before analysis, it was redissolved in $100 \mu \mathrm{l}$ deionised water containing 10\% heptafluorobutyric acid (HFBA) and filtered through a $0.2 \mu \mathrm{m}$ filter (Gelman Sciences, Northampton) into high performance liquid chromatography (HPLC) ampoules. 
The samples were analysed by reverse phase HPLC using a modification of the method of Eyre et al. ${ }^{22}$ Each sample was chromatographed using a $150 \times 4.6 \mathrm{~mm}$ Spherisorb S3 ODS column (Phase Separations Ltd, Deeside, Clwyd, North Wales). The cross linking amino acids hydroxylysyl pyridinoline and pentosidine were eluted isocratically using $18 \%$ and $30 \%$ acetonitrile respectively in deionised water containing $0.05 \mathrm{M}$ HFBA.
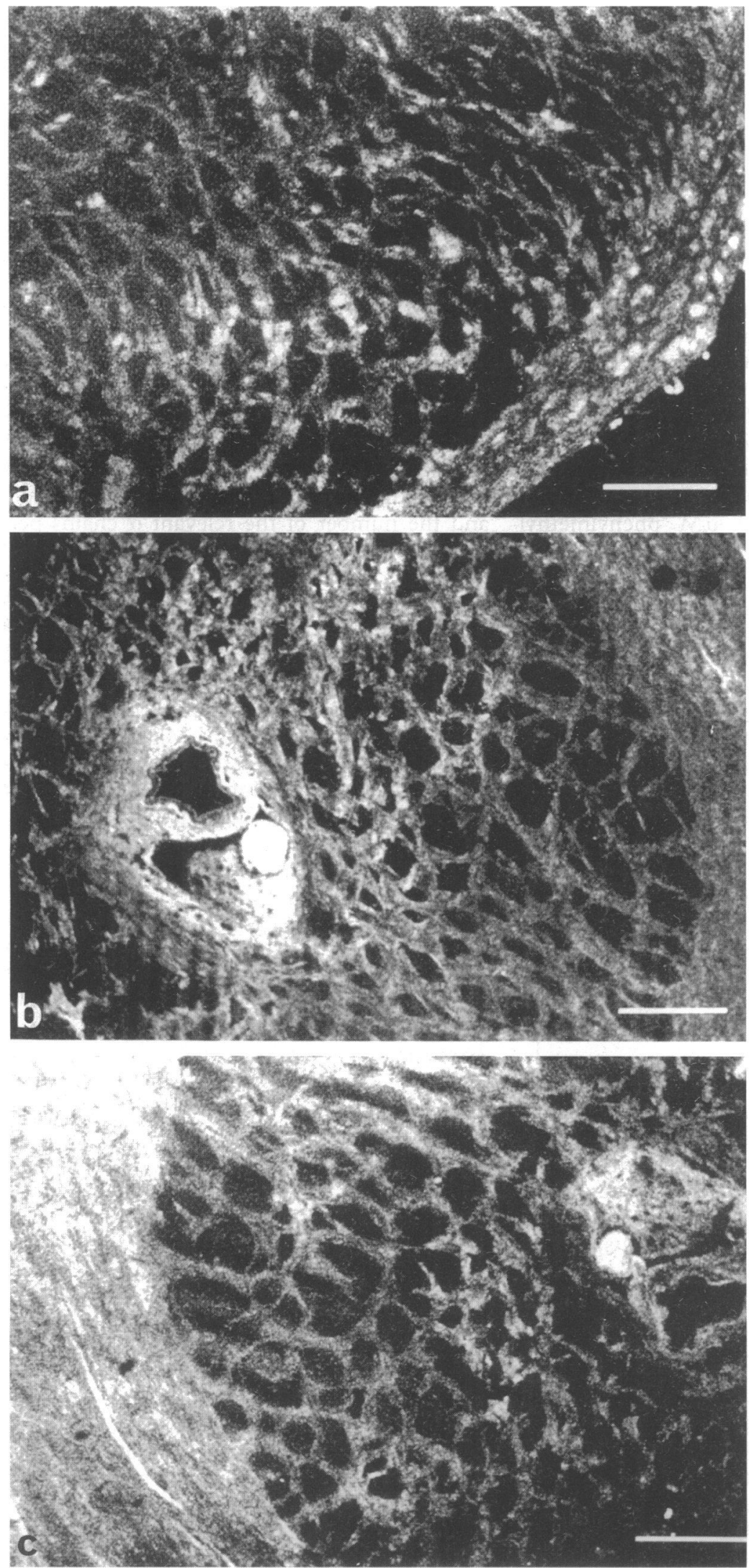

The natural fluorescence of pentosidine and hydroxylysylpyrinoline was detected using a Perkin-Elmer fluorimeter at excitation/emission wavelength maxima of $335 / 385 \mathrm{~nm}$ and $295 / 405 \mathrm{~nm}$ respectively.

\section{STATISTICAL ANALYSIS}

Spearmen's rank correlation was used to test the tendency of values of $\mathrm{Y}$ (that is, \% collagen, \% type III collagen, \% solubilisation, hydroxylysyl pyridinoline, pentosidine) to increase (or decrease) as values of $\mathrm{X}$ (age) increase.

\section{Results}

ISOLATION OF LAMINA CRIBROSA

\section{Immunolocalisation of collagen types}

The distribution of collagen related immunofluorescence detected by the conventional epifluorescence microscopy was similar to that detected by the confocal laser scanning microscope. The lamina cribrosa stained positive when probed with antibodies against human collagen types I, III, IV, V, and VI as demonstrated in Figures $1 \mathrm{a}-\mathrm{e}$, and negative against human type II collagen antibody (not shown).

Immunofluorescence for types I (Fig 1a), III (Fig 1b), and V (Fig 1c) collagen appeared to codistribute in the walls of the central retinal vessels, the peripheral sclera surrounding the lamina cribrosa, and in the connective tissue surrounding the nerve fibre bundles. Strong staining for type III collagen was observed surrounding the central retinal vessels in contrast with the more limited staining of type $\mathrm{V}$ collagen in this location. This is consistent with other published work which suggests that individual banded collagen fibres of most noncartilaginous tissues contain both collagen types I and III. ${ }^{23}$ Collagen types I and V are also thought to form heterotypic fibres in some tissues - for example, collagen type $\mathrm{V}$ is thought to have a role, in part, in the regulation of collagen fibril diameter in the cornea. ${ }^{24}$

Type IV collagen staining differed from that of the fibrillar collagens; negative for the peripheral sclera, positive for the basement membranes of the astroglial cells surrounding the nerve fibre bundles and the endothelial cells lining the central retinal vessels (Fig 1d). More sparse staining was identified within the cribriform plates (Fig 1d). Type VI collagen was identified throughout the lamina cribrosa, in the peripheral sclera and in the walls of the central retinal vessels (Fig 1e).

\section{Dissection procedure}

Owing to the presence of several collagen types in the central retinal vessels and sclera, confirmed by our immunohistochemical results, a method was developed to dissect the lamina cribrosa away from these tissues and the underlying optic nerve.

Portions of the sclera and optic nerve were 

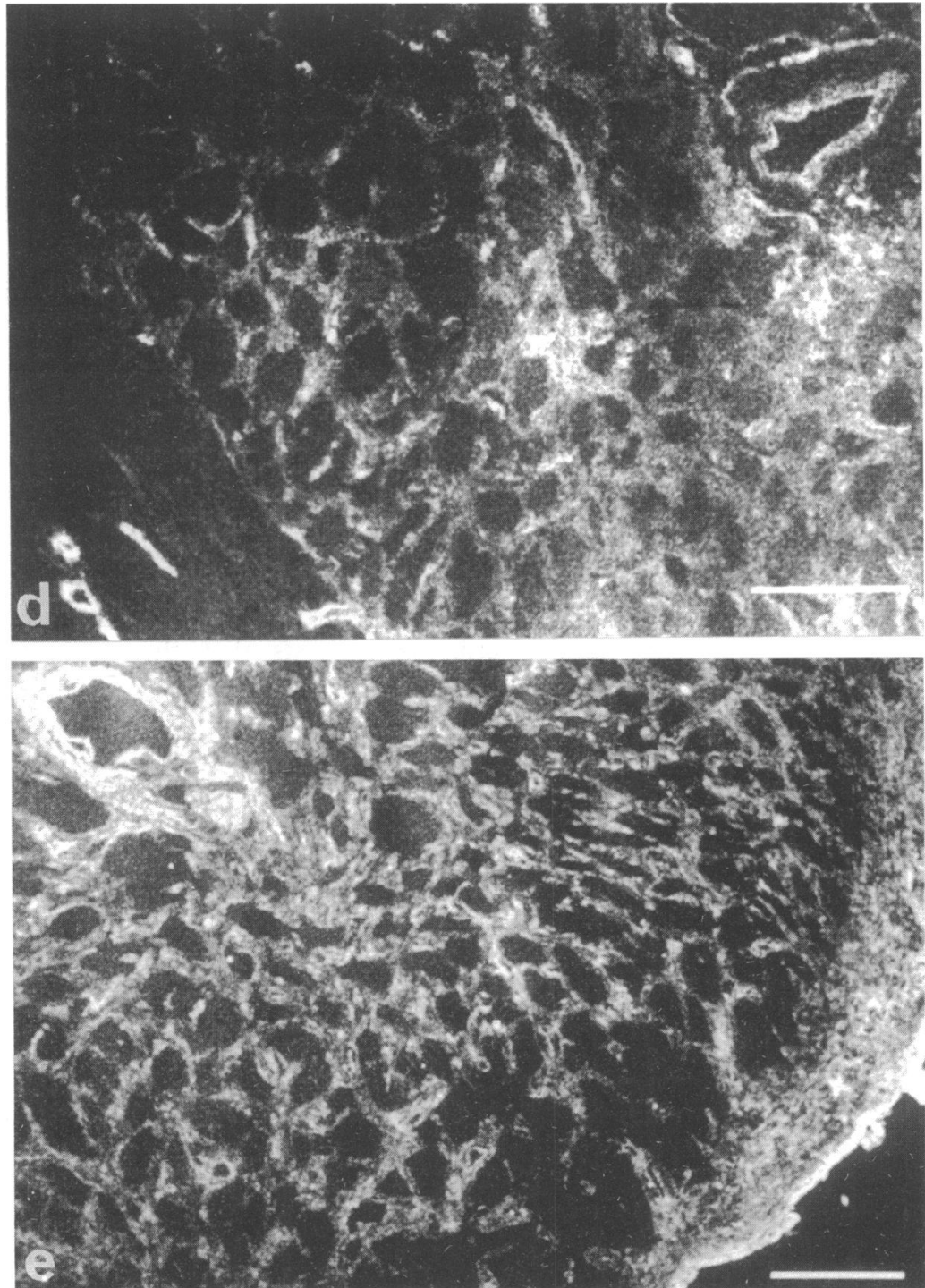

Figure 1 Immunofluorescent staining of cross sections through the human lamina cribrosa. The sections were probed with primary antibodies against collagen types $I(a), I I I(b), V$ (c), IV (d), and VI (e), and then linked to a FITC conjugate. Bar=100 $\mu \mathrm{m}$.

separated from the rest of the eye. Using a Zeiss DV4 dissecting microscope the remaining retinal and choroidal tissue was removed from the front of the optic disc. Glial tissue was dissected from the front of the lamina cribrosa by cutting, at the same level as the sclera, around the circumference of the optic disc and then carefully teasing it away. The lamina cribrosa was then isolated from surrounding ocular tissues by severing the optic nerve just behind the lamina cribrosa and detaching it carefully from the surrounding sclera. To avoid analysis of any collagen from the central retinal vessels, these vessels were carefully removed (Figs $2 \mathrm{a}$ and $2 \mathrm{~b}$ ). Figures $2 \mathrm{a}$ and $2 \mathrm{~b}$ illustrates a dissected lamina cribrosa with central retinal vessels intact and a dissected lamina cribrosa with central retinal vessels removed respectively. Laminae cribrosae similar to the one in Figure $2 b$ were used for all biochemical analyses.

COLLAGEN CONTENT

An increase in the total collagen present in the lamina cribrosa as a percentage of dry tissue weight was demonstrated with age (Fig 3).
Collagen content increased from $20 \%$ in young laminae cribrosae to about $50 \%$ in the elderly (Spearman's rank correlation $r_{\mathrm{s}}=0.589$, $\mathrm{p}<0.001, \mathrm{n}=46$ ).

TYPE I AND TYPE III COLLAGEN

The proportion of type III collagen as a percentage of total fibrous collagen (that is, type I+type III collagen) demonstrated an agerelated decrease from $23 \%$ at the age of 9 weeks to $3 \%$ at 92 years (Fig $4, r_{\mathrm{s}}=0.878$, $\mathrm{p}<0.001, \mathrm{n}=38$ ). The decrease was apparently linear with age although in the older age range, the level of type III collagen was approaching the lower limits of detection and therefore subject to greater inaccuracy.

SOLUBILISATION OF COLLAGEN

Solubility of collagen, by $\mathrm{CNBr}$ digestion, decreased from $100 \%$ in laminae cribrosae under the age of 1 year to $40 \%$ in laminae cribrosae in the seventh decade of life (Fig 5, $\left.r_{\mathrm{s}}=-0.916, \mathrm{p}=0.0001, \mathrm{n}=58\right)$. The degree of solubility was unaffected by reduction of oxidised methionine residues using DTT before $\mathrm{CNBr}$ digestion.

\section{COLLAGEN CROSS LINKS}

Hydroxylysylpyridinoline (HP) is a mature collagen cross link in many tissues. Low levels of HP in laminae cribrosae of all ages demonstrated no significant variation, but persisted throughout life, with an average of 0.1 mole of cross link per mole of collagen (Fig 6, $r_{\mathrm{s}}=0.272$, not significant, $\mathrm{p}=0 \cdot 146, \mathrm{n}=30$ ). Pentosidine, a collagen cross link formed by non-enzymatic glycosylation, demonstrated a linear age-related increase in the lamina cribrosa (Fig 7, $r_{\mathrm{s}}=0.781, \mathrm{p}<0.001, \mathrm{n}=31$ ).

\section{Discussion}

Immunofluorescent localisation of the different collagens enabled us to ascertain the distribution and location of collagen in the sclera and in and around the central retinal vessels, so that these regions could be identified and separated from the lamina cribrosa. Type I, III, and $\mathrm{V}$ collagens appeared to codistribute within the cribriform plates and also in the walls of the central retinal vessels. Type $\mathrm{V}$ is thought to act as an anchoring network by binding basement membranes to interstitial collagens. ${ }^{15}$ Type IV collagen delineated the basement membranes of the central retinal vessels and those of the astrocytes along the margins of the cribriform plates and sparsely within their cores. Type VI collagen, believed to play a role in maintaining the integrity of a tissue by acting as a flexible anchor connecting collagen fibrils and cells, ${ }^{16}$ was also identified within the plates. Type II collagen was undetected throughout the lamina cribrosa. The distribution of laminar collagens is consistent with previous immunolocalisation studies. ${ }^{9} 10$ Type IV collagen is usually considered a component of basement membranes. However, 

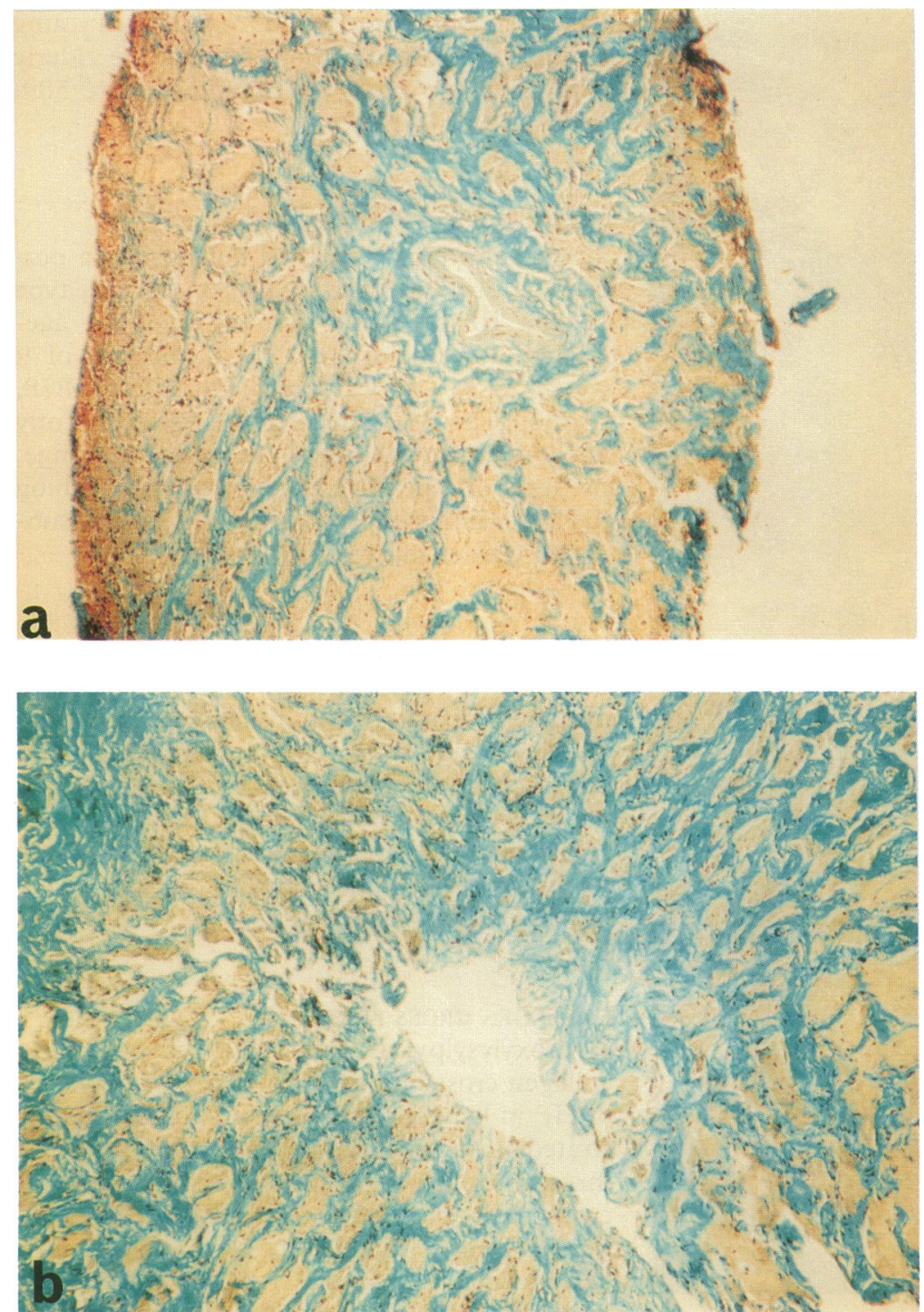

Figure 2 Cross sections through a dissected human lamina cribrosa (a) and a dissected lamina cribrosa after the central retinal vessels have been removed (b). Sections were stained using Masson's trichrome green which is a generalised connective tissue stain. Magnification $\times 78$.

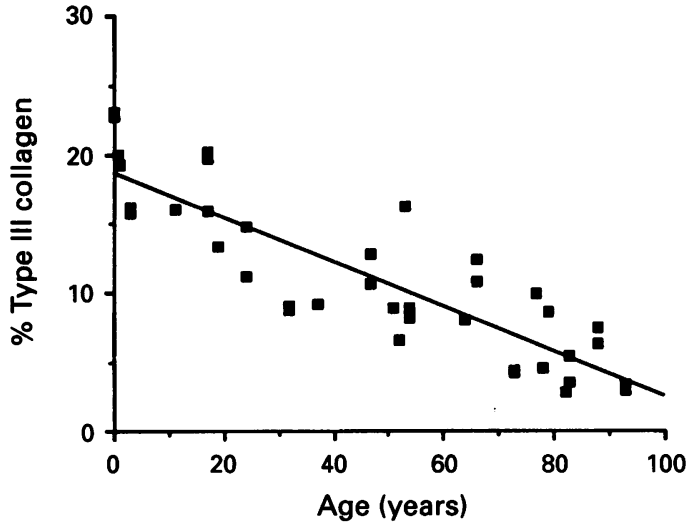

Figure 4 Percentage type III collagen, as a proportion of total fibrous collagen, present in the human lamina cribrosa plotted as a function of age.

collagens within the extracellular matrix of the lamina cribrosa suggests that the different types work together to maintain its mechanical integrity.

The above immunohistochemical results reveal a large amount of collagen staining in the sclera and central retinal vessels. Thus a routine method to isolate the lamina cribrosa from immediate ocular tissues (Figs 2a and b) has been successfully developed. The careful removal of the central retinal vessels (Fig 2b) and surrounding sclera avoided any misleading biochemical analyses derived from collagen in these structures.

The connective tissue plates of the lamina cribrosa provide essential support to the retinal ganglion cell axons as they leave the retina to enter the optic nerve. Collagen contributes to the tensile properties of a tissue depending on which collagen types are present. In the aging lamina cribrosa an increase in total collagen as a percentage of dry tissue weight is consistent with earlier published work ${ }^{11}$ which describes increases in collagen related immunofluorescence of types I, III, and IV collagen, which accompanies an age-related thickening of the cribriform plates. ${ }^{1126}$ This increase in collagen and increase in thickness of the cribriform plates may result in a decrease in the overall flexibility of the lamina cribrosa with age.

Types I and III collagen are the major types in the lamina cribrosa. ${ }^{26}$ These types codistribute in many tissues representing $95 \%$ of the

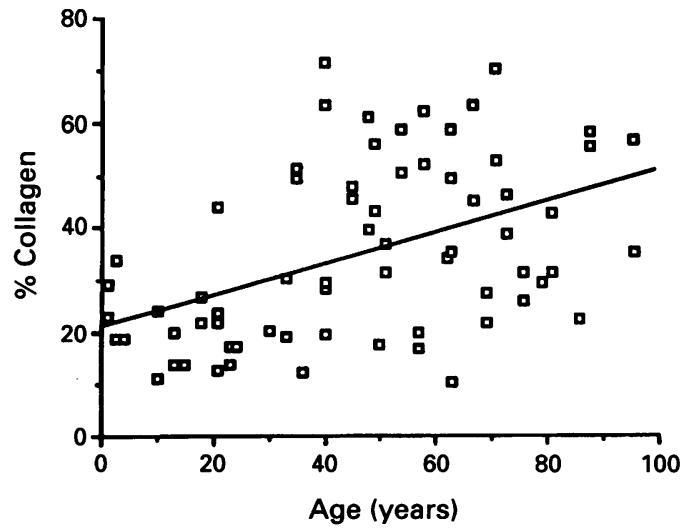

Figure 3 Total collagen in the human lamina cribrosa. Total collagen was determined by hydroxyproline analysis, and expressed as a percentage of dry tissue weight, plotted as a function of age.

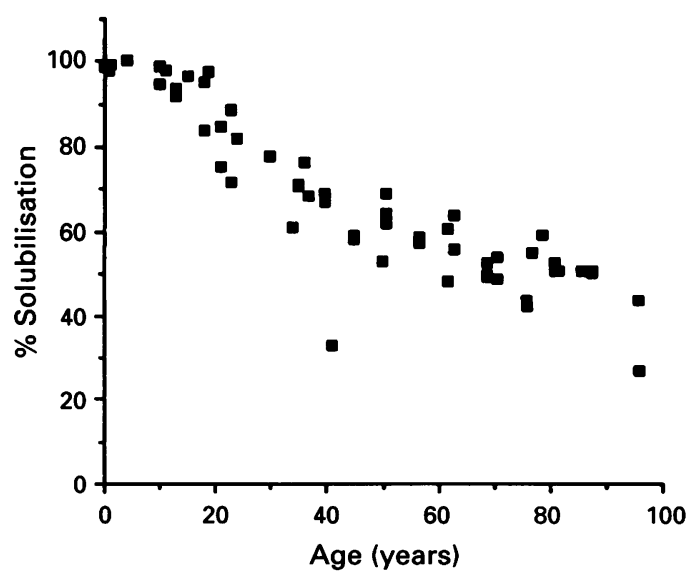

Figure 5 Solubility of collagen by cyanogen bromide digestion in the aging human lamina cribrosa. 


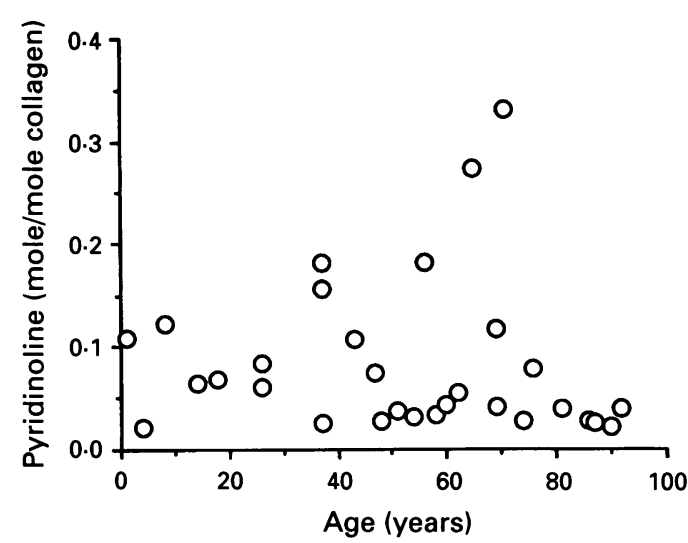

Figure 6 Hydroxylysyl pyridinoline (mole of cross link per mole of collagen) levels in the aging human lamina cribrosa.

total fibrillar collagen and are responsible for the tensile properties of the tissues they constitute. Diameters of type I collagen fibrils are believed to be regulated by the presence of type III collagen and also pNIII collagen (type III collagen with the $\mathrm{N}$-terminus propeptide still attached). ${ }^{27}$ Type I fibrils are larger and less flexible than those formed by mixtures of types I and III or type III alone. Therefore it is not surprising that type III collagen is usually found in extensible, flexible tissues such as in fetal tissue, lungs, skin, and blood vessels. ${ }^{28}$

In the lamina cribrosa, type III as a percentage of total fibrous collagen decreased linearly from $23 \%$ to $3 \%$ between the ages of 3 weeks and 96 years. The altered type III to I ratio agrees with the suggestion by Morrison et al that the predominance of type III in immature laminae cribrosae is replaced by an increase in type I collagen in adults. ${ }^{26}$ In contrast, Hernandez et al ${ }^{11}$ demonstrated an age-related increase in labelling for type III collagen, with very little labelling in young laminae cribrosae. This may be due to masking of the type III collagen epitopes recognised by this antibody, by other constituents of the extracellular matrix in young laminae cribrosae, such as proteoglycans. Indeed, Sawaguchi et $a l^{29}$ have demonstrated an age-related decline in chondroitin/dermatan sulphate proteoglycans and in our laboratory, biochemical analyses have revealed a higher level of sulphated glycosaminoglycans in young human laminae cribrosae especially those aged between 0 and 20 years (unpublished data).

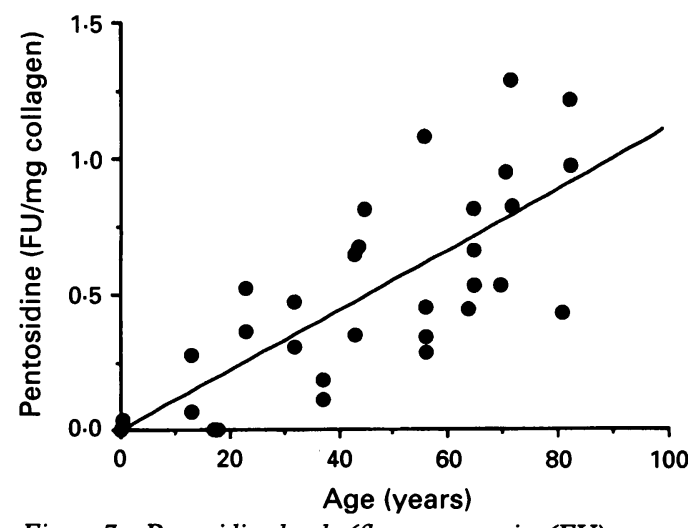

Figure 7 Pentosidine levels (fluorescent units (FU) per milligram of collagen) in the aging human lamina cribrosa.
The localisation of mRNA for collagen types I and III has been demonstrated in an age range of optic nerve heads implying that these collagen types are synthesised throughout life. ${ }^{3031}$ Thus the age-related decrease in the proportion of type III collagen suggests that the rate of synthesis and/or degradation of these two collagen types differs. The decrease in type III collagen implies a reduction in mechanical compliance of the aging lamina cribrosa. This may, in part, explain the reversibility of optic disc cupping in children, which itself suggests the presence of a resilient structure. ${ }^{32-34}$

The decrease in solubilisation of collagen by $\mathrm{CNBr}$ digestion implies that the collagenous matrix of the lamina cribrosa is altered during the aging process so that complete solubilisation is prevented. $\mathrm{CNBr}$ cleaves collagen into peptides by attacking the C-terminus of methionine residues in the reduced state. Prior reduction of methionine residues using $5 \mathrm{mM}$ DTT did not increase the degree of solubilisation indicating that the decreased collagen solubility is not due to an age-related increase in oxidised methionine and therefore may be attributed to increased cross linking.

Hydroxylysyl pyridinoline (HP) is a mature collagen cross link derived from those initiated by the enzyme lysyl oxidase. ${ }^{35}$ Other mature collagen cross links include lysyl pyridinoline, which is not present in the lamina cribrosa, and histidinohydroxylysinonorleucine. Lysyl pyridinoline is found mainly in bone and dentine collagen, whereas the latter is present in skin, sclera, and cornea. ${ }^{35} \mathrm{We}$ were unable to identify the presence of histidinohydroxylysinonorleucine and the immature collagen cross links: dehydrohydroxylysinonorleucine and hydroxylysino-keto-norleucine because of the small sample size, which is a major limitation for analysis. The natural fluorescence of the pyridinolines and pentosidines increases the level of sensitivity for analysis which facilitates their detection.

Levels of HP demonstrated no significant variation with age in laminae cribrosae, but persisted throughout life, indicating that any age-related changes in solubility or physical properties are unlikely to be attributed to levels of HP alone. The levels of HP in the lamina cribrosa were relatively low, suggesting that other mature collagen cross links may be present.

In the human lamina cribrosa, an agerelated increase in pentosidine was demonstrated. Pentosidine is at present the only advanced Maillard product which has been fully characterised. It is a pentose derived fluorescent cross link formed between lysine and arginine residues in collagen. ${ }^{36}$ Pentosidine has been linked to an age-related stiffening of collagen-rich tissues such as lungs, arteries, and joints and is present in increased levels in age and diabetes related diseases like hypertension, emphysema, decreased joint mobility, and the ability to fight infections. ${ }^{37}$ Therefore the rise in pentosidine in the aging lamina cribrosa suggests a stiffening or increased rigidity of the lattice-like connective 
tissue structure with age. Other evidence in support of this includes the in vitro incubation of rat tail tendon and lens capsule with glucose, which has demonstrated an increased mechanical stability as determined by an increased thermal rupture time and thermal denaturation temperature, respectively. ${ }^{38}$ The mechanical stiffness of collagen has been shown to increase in experimental diabetes. ${ }^{39}$ In both these cases the increased stability is thought to be a result of an increase in advanced glycation products.

In the lamina cribrosa it is not known if the very small amount of pentosidine present will have any significant effect on its mechanical properties. Investigations by Dyer et $a l^{40}$ and Sell and Monnier ${ }^{36} 37$ confirm the presence of pentosidine in other tissues in trace quantities. A strong relation between increases in pentosidine and collagen linked fluorescence in aging suggests that, despite its low concentration in tissues, pentosidine may be a generalised marker of accelerated tissue modifications and protein damage by the advanced glycation/Maillard reaction. The apparent increase in cross linking, suggested by the agerelated decrease in collagen solubility, may possibly be explained by the rise in pentosidine.

In conclusion, biochemical alterations in the collagenous matrix of the aging lamina cribrosa - that is, age-related increases in total collagen and pentosidine and a decrease in type III collagen, are consistent with our observations of the change in mechanical properties with age, which indicate a reduced compliance and increased rigidity. ${ }^{4142}$ In infants, glaucomatous optic disc cupping is reversible, ${ }^{32-34}$ whereas in adults the optic disc cupping is rarely reversible. ${ }^{43}$ This is consistent with our biochemical findings in suggesting that the young lamina cribrosa is a more resilient and compliant structure.

Misalignment of the cribriform plates of the lamina cribrosa occurs in glaucomatous optic neuropathy. The more rigid plates of the elderly will create larger forces on the nerve fibre bundles than those in the compliant plates of the young lamina cribrosa, thus increasing the susceptibility of axons to damage.

Further investigation is needed to determine whether the connective tissue in the glaucomatous lamina cribrosa ${ }^{44}$ is abnormal in nature or a result of injury or pressure induced remodelling of the extracellular matrix.

This project was supported by the Guide Dogs for the Blind Association and the National Eye Research Centre. The authors would also like to thank the Bristol Eye Bank for the provision of samples.

1 Levene RZ. Low tension glaucoma: a critical review and new material. Surv Ophthalmol 1980; 24: 621-64.

2 Fechtner RD, Weinreb RN. Mechanisms of optic nerve damage in primary open-angle glaucoma. Surv Ophthalmol 1994; 39: 23-42.

3 Sommer A. Intraocular pressure and glaucoma. $\mathrm{Am} f$ Ophthalmol 1989; 107: 186-8.

4 Quigley HA, Addicks EM. Regional differences in the structure of the lamina cribrosa and their relation to glaucomatous optic nerve damage. Arch Ophthalmol 1981; 99: $137-43$.

5 Radius RL, Gonzales M. Anatomy of the lamina cribrosa in human eyes. Arch Ophthalmol 1981; 99: 2159-62.
6 Quigley HA, Addicks EM, Green WR, Maumenee AE. Optic nerve damage in glaucoma: II The site of injury and susceptibility to damage. Arch Ophthalmol 1981; 99: 635-49.

7 Quigley HA, Hohman RM, Addicks EM, Massof RW, Green WR. Morphological changes in the lamina cribrosa correlated with neural loss in open-angle glaucoma. Am $\mathcal{f}$ Ophthalmol 1983; 95: 673-91.

8 Miller KN, Quigley HA. The clinical appearance of the lamina cribrosa as a function of the extent of glaucomatous optic nerve damage. Ophthalmology 1988; 95: 135-8.

9 Hernandez MR, Luo XX, Igoe F, Neufeld AH. Extracellular matrix of the human lamina cribrosa. Am $\mathcal{f}$ Ophthalmol 1987; 104: 567-76.

10 Goldbaum MH, Jeng S, Logemann R, Weinreb RN. The extracellular matrix of the human optic nerve. Arch Ophthalmol 1989; 107: 1225-31.

11 Hernandez MR, Luo XX, Andrzejewska W, Neufeld AH. Age-related changes in the extracellular matrix of the human optic nerve head. Am $\mathcal{F}$ Ophthalmol 1989; 107: 476-84.

12 Hernandez MR, Andrzejewska WM, Neufeld AH. Changes in the extracellular matrix of the human optic nerve head in chronic open-angle glaucoma. Am 7 Ophthalmol 1990; 109: $180-8$.

13 Quigley HA, Dorman-Pease ME, Brown AE. Quantitative study of collagen and elastin of the optic nerve head and sclera in human and experimental glaucoma. Curr Eye Res 1991; 10: 877-88.

14 Hernandez MR. Ultrastructural immunocytochemistry of elastin in the human lamina cribrosa: changes in elastic fibres in chronic open-angle glaucoma. Invest Ophthalmol Vis Sci 1992; 33: 2891-900.

15 Modesti A, Kalebic T, Scarpa S, Togo S, Grotendorts G, Liotta LA, Triche TJ. Type V collagen in human amnion is a $12 \mathrm{~nm}$ fibrillar component of the pericellular interstitium. Eur f Cell Biol 1984; 35: 246-55.

16 Keene DR, Engvall E, Glanville RW. Ultrastructure of type VI collagen in human skin and cartilage suggests an anchoring function for this filamentous network. f Cell Biol 1988; 107: 1995-2006.

17 Anderson DR. Glaucoma: the damage caused by pressure. XIVI Edward Jackson Memorial Lecture. Am f Ophthalmol 1989; 108: 485-95.

18 Bannister DW, Burns AB. Adaptation of the Bergman and Loxley technique for hydroxyproline determination to the autoanalyser and its use in determining plasma hydroxyproline in the domestic fowl. Analyst 1970; 95: 596-600.

19 Light ND. Estimation of types I and III collagens in whole tissue by quantitation of $\mathrm{CNBr}$ peptides on SDS-polytissue by quantitation of CNBr peptides on SDS-poly-

20 Laemmli UK. Cleavage of structural proteins during assembly of the head of bacteriophage T4. Nature (London) 1970; 227: 680-2

21 Lovell CR, Smolenski KA, Duance VC, Light ND, Young S, Dyson M. Type I and III collagen content and fibre distribution in ' normal human skin during ageing. Br f Dermatol 1987; 117: 419-28.

22 Eyre DR, Koob J, Van Ness KP. Quantitation of hydroxypyridinium cross-links in collagen by high performance liquid chromatography. Anal Biochem 1984; 137: 380-8.

23 Keene DR, Sakai LY, Bachinger HP, Burgeson RE. Type III collagen can be present on banded fibrils regardless of III collagen can be present on banded fibrils rega

24 Birk DE, Fitch JM, Babiarz JP, Linsenmayer TF. Collagen type $I$ and type $V$ are present in the same fibril in the avian corneal stroma. F Cell Biol 1988; 106: 999-1008.

25 Malak TM, Ockleford CD, Bell SC, Dalgleisch R, Bright T, MacVicar J. Confocal immunofluorescent localisation of collagen types I, III, IV, V and VI and their ultrastructural organisation in human foetal membranes. Placenta 1993; 14: $385-406$.

26 Morrison JC, Jerdan JA, Dorman ME, Quigley HA Structural proteins of the neonatal and adult lamina Structural proteins of the neonatal and ad
cribrosa. Arch Ophthalmol 1989; 107: 1220-4.

27 Romanic AM, Adachi E, Kadler KE, Hojima Y, Prockop DJ. Copolymerization of $\mathrm{pN}$ collagen and collagen I f Biol Chem 1990; 265: 12703-10.

28 Montes GS, Bezerra MSF, Junqueira LCU. Collagen distribution in tissues. In: Ruggeri A, Motta PM, eds Ultrastructure of the connective tissue matrix. Boston Martinus Nijhoff, 1984: 65-88.

29 Sawaguchi. Age-related changes of sulphated proteoglycans in the human lamina cribrosa. Curr Eye Res 1993; 12: 685-92.

$30 \mathrm{Ye} \mathrm{H}$, Yang J, Hernandez MR. Localisation of collagen type III mRNA in normal human optic nerve heads. Exp Eye Res 1994; 58: 53-63.

31 Hernandez MR, Hanley NM, Neufeld AH. Localisation of collagen types I and IV mRNAs in human optic nerve head by in situ hybridisation. Invest Ophthalmol Vis $S c$ 1991; 32: 2169-77.

32 Schaffer RN, Hetherington J. The glaucomatous disc in infants: a suggested hypothesis for disc cupping. Trans $A m$ Acad Ophthalmol Otolaryngol 1969; 73: 929-35.

33 Quigley HA. The pathogenesis of reversible cupping in congenital glaucoma. Am $\mathcal{Y}$ Ophthalmol 1977; 84: 358-70.

34 Quigley HA. Childhood glaucoma: results with trabeculotomy and study of reversible cupping. Ophthalmology 1982; 89: 219-26.

35 Eyre DR, Paz MA, Gallop PM. Cross-linking in collagen and elastin. Annu Rev Biochem 1984; 53: 717-48. 
36 Sell DR, Monnier VM. Structure elucidation of a senescence cross-link from human extracellular matrix - implication of pentoses in the ageing process. $\mathcal{F} \mathrm{Biol} \mathrm{Chem} \mathrm{1989;}$ 264: 21597-602.

37 Sell DR, Monnier VM. End-stage renal disease and diabetes catalyse the formation of a pentose-derived cross-link catalyse the formation of a pentose-derived cross-link
from ageing human collagen. $\mathcal{f}$ Clin Invest 1990; 85: from agei.

38 Bailey AJ, Kent C. Non-enzymatic glycosylation of fibrous and basement membrane collagens. In: Baynes JS, Monnier VM, eds. The Maillard reaction in ageing, diabetes, and nutrition. New York: Alan R Liss, 1989: 109-22.

39 Andreassen TT, Seyer-Hansen K, Bailey AJ. Thermal stability, mechanical properties and reducible cross-links of rat tail tendon in experimental diabetes. Biochim Biophys Acta 1981; 677: 313-7.
40 Dyer DG, Blackledge JA, Thorpe SR, Baynes JW. Formation of pentosidine during nonenzymatic browning of proteins by glucose. F Biol Chem 1991; 266: 11654-60.

41 Albon J, Purslow PP, Duance VC, Easty DL, Karwatowski WSS. Mechanical compliance of the ageing human lamina cribrosa. Invest Ophthalmol Vis Sci 1994; 35 (suppl): 1645 .

42 Albon J, Purslow PP, Karwatowski WSS. Age-related compliance of the lamina cribrosa in human eyes. $f$ Biomechanics 1994; 27: 823 .

43 Lusky M, Morsman D, Weinreb RN. Effect of intraocular pressure on optic nerve head topography. Curr Opin Ophthalmol 1993; 4: 40-4.

44 Hernandez MR, Ye H. Glaucoma: changes in extracellular matrix in the optic nerve head. Ann Med 1993; 25: 309-15.

\section{History of ophthalmology}

\section{The discovery of accommodation}

The story of accommodation was pieced together over three centuries, by workers using only the most basic investigative tools. Knives, paper, muslin, candles, and blunt instruments are the only materials used in the experiments described below.

To set the scene, Aristotle and Galen had dissected animal and human eyes. From their drawings the basic anatomy of the globe and lens were common knowledge. Telescopes and the need to focus them had also been discovered. From this arises a first principle of optics: to vary focus, either the dioptric power or the length of an eyeball/telescope must change.

In 1619 , Scheiner published the results of an experiment proving that the eyes' dioptric power changes. Observing a near object through a paper screen with two tiny holes close together, the object is single. But focusing on any far object, the near object becomes double. Although Scheiner's conclusion was valid, it was disputed.

Some believed that the eyeballs focused by changing their length, elongating and shortening within the socket at need. This was refuted beautifully by Thomas Young in 1801. Young noted that a blunt object pressed on the posterior part of his eyeball produced a scotoma, which increased in size on increasing the pressure. Doing this during accommodation, Young saw no increase in scotoma, and concluded that the bar did not press more deeply on the back of the globe, therefore the eye could not have lengthened. Because he published his results in Philosophical Transactions, ophthalmologists took little notice.

Others accepted that the lens was involved in accommodation, but thought it changed its position within the globe. Many denied that accommodation took place at all. Haller, in 1743 , asserted that the pupillary constriction seen on converging for near objects in some way altered the depth of focus, and that no other explanation was required.

The controversy persisted. Donders, publishing his book on accommodation around 1860 , found it necessary to stress his own version of Scheiner's experiment. This involved holding a veil some inches from the eye, and an open book at greater distance. Either the texture of the veil, or the print could be seen accu- rately, but not both at once. 'Ergo, accommodation must exist,' reasoned Donders.

To appreciate the first proof that it resides in a change of form in the lens, an experiment performed in 1823 must be considered. Purkinje looked at the reflection of candlelight in someone's eyes and noted three reflected images. One was large, one small, and one inverted, and he correctly attributed them to reflection off the cornea, and the anterior and posterior surfaces of the lens.

This was initially applied to the diagnosis of cataract, but in 1849, Langenbeck realised that the images reflected from the lens could be used to investigate accommodation. Langenbeck published the right result in the wrong journal and was ignored. Thus it was left to Cramer to promulgate the following findings - that, holding a flame in front of an eye, the image reflected from the anterior lens surface becomes smaller when the subject accommodates. For the image to become smaller, the surface must increase in convexity. Therefore, the lens does change shape in accommodation. Helmholtz went a step further in noting that the image reflected from the posterior lens surface also changes, therefore that surface also increases in convexity.

The controversy lasted into the 1960 s, when textbooks quoted two rival theories of accommodation. Helmholtz believed that ciliary muscle contraction relaxed the zonule, whereupon the elastic lens became more curved. Tscherning believed that contraction tensed the zonule, which compressed the lens capsule to increase its curvature. Both theories depended on the lens being elastic, which was accepted by Donders in 1860, and confirmed in rabbits' eyes by Kikawa in 1963.

The acceptance of Helmholtz's theory over the next years ends the story, but the initial discoveries (that accommodation exists, involves the lens, and that the lens changes shape), made with the aid of such simple materials, make a very impressive tale of deductive reasoning.

F ROMAN

Adler FC. Physiology of the eye. St Louis: Mosby, 1965: 280-91. Davison H. The physiology of the eye. London: Churchill, 1949:

230-43.
Donders FC. Accommodation on the eye. Quoted in: Glendening L. Source book of medical history. New York: Henry Schuman, 1942. 